\title{
POINT-JOINT COORDINATE FORMULATION FOR THE DYNAMIC ANALYSIS OF GENERALISED PLANAR LINKAGES
}

\author{
HAZEM ALI ATTIA ${ }^{1}$
}

(Received 2 January 2003; revised 26 April 2004)

\begin{abstract}
This paper presents a two-step formulation for the dynamic analysis of generalised planar linkages. First, a rigid body is replaced by a dynamically equivalent constrained system of particles and Newton's second law is used to study the motion of the particles without introducing any rotational coordinates. The translational motion of the constrained particles represents the general motion of the rigid body both translationally and rotationally. The simplicity and the absence of any rotational coordinates from the final form of the equations of motion are considered the main advantages of this formulation. A velocity transformation is then used to transform the equations of motion to a reduced set in terms of selected relative joint variables. For an open-chain, this process automatically eliminates all of the non-working constraint forces and leads to efficient integration of the equations of motion. For a closed-chain, suitable joints should be cut and some cut-joint constraint equations should be included. An example of a closed-chain is used to demonstrate the generality and efficiency of the proposed method.
\end{abstract}

\section{Introduction}

In recent years, there have been many attempts to develop efficient methods for generating the equations of motion for multibody systems [8-11]. These methods can be divided into two main approaches depending on the type of coordinates chosen. In the first approach, the equations of motion are formulated using relative joint coordinates $[2,7,12]$. For an open-chain all the generalised coordinates are independent, while for a closed-chain a minimum number of dependent coordinates should be defined. This leads to an efficient solution and integration of the equations of motion. However, in many applications, this approach leads to a relatively complex recursive formulation based on the loop closure equations and it is also difficult to incorporate general constraints and forcing functions.

\footnotetext{
${ }^{1}$ Department of Mathematics, College of Science, Al-Qasseem University, P.O. Box 237, Buraidah 81999, KSA; e-mail: ah1113@yahoo.com.

(C) Australian Mathematical Society 2005, Serial-fee code 1446-1811/05
} 
The second approach uses the absolute coordinates that describe the location and orientation of the bodies in the system with respect to an inertial reference frame $[4,6]$. In this approach each body has identical coordinate representation and the constraint equations are easily formulated for each joint. A main disadvantage of this method is the large number of mixed differential and algebraic equations which leads to inefficient solution and integration of the equations of motion. Another approach for multibody dynamics modelling uses both coordinate types $[3,5]$. The equations of motion are first formulated in terms of the absolute coordinates and then transformed to relative joint coordinates through the use of a velocity transformation matrix.

In this study, the rectangular Cartesian coordinates of an equivalent constrained system of particles are used to define the position of the system initially. The mass associated with each particle is determined as a function of the inertia characteristics of each body. The differential equations of motion are derived by applying Newton's second law to study the translational motion of the particles without introducing any rotational coordinates. The equations of motion are then transformed to a reduced set in terms of a selected set of relative joint variables using a velocity transformation matrix which allows efficient solution and integration of the equations of motion without loss of generality. The dynamic analysis of a closed-chain is chosen to demonstrate the efficiency and generality of the formulation.

\section{Construction of the equivalent system of particles}

A rigid body and its dynamically equivalent system of particles should have the same mass, the same position of centre of mass and the same moment of inertia about an axis perpendicular to its plane which results in four conditions for the planar case. It should be noted that the equivalence is fulfilled without any approximations. Since these physical quantities (mass, centre of mass, polar moment of inertia) are the only constitutive properties that control the motion of both systems (the rigid body and its dynamical equivalent system of particles), then the construction of the equivalent system of particles can be done using the four conditions only once at the beginning of the motion. The choice of three particles with nine unknowns (three masses + six coordinates) satisfies the four conditions but it has the disadvantage that it does not give the freedom to choose all the particles in important places for mechanisms. The choice of four particles gives a unique solution for the four conditions in four unknown masses. At the same time it has the advantage that it allows freedom in choosing the locations of all particles in important places for mechanisms. The representation of the rigid body by equivalent particles and the freedom in locating these particles has an interesting application in flexible multibody systems when a deformable body is connected to a rigid body described by a set of particles. Then one or more particles 


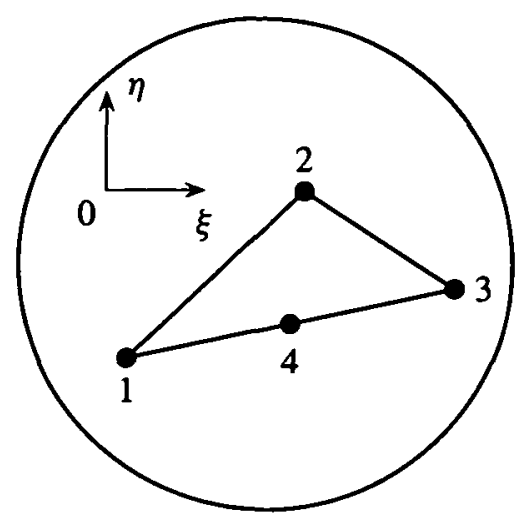

FIGURE 1. The rigid body with its equivalent system of three particles.

of the rigid body can coincide with one or more nodes of the deformable body in order to describe the kinematical joint between the two bodies. This is a much simpler process than when the rigid body is described by a set of translational and rotational coordinates. Choosing more than four particles will have the same advantage as the choice of four particles but with the introduction of extra free unknowns that must be arbitrarily chosen. Also, since more particles have been used, this will lead to more complicated equations of motion. Therefore the choice of four particles seems to be the most suitable. In this case, as shown in Figure 1, four particles are chosen to represent the rigid body in order to obtain a linear system of four equations in four unknown masses. Then the coordinates of the four particles become free variables and can be arbitrarily chosen to achieve additional requirements. Three particles, 1 , 2 and 3, are located arbitrarily, while the remaining fourth particle is conveniently located at the mid-point of the line joining particles 1 and 3. By this arrangement, the four conditions are written as:

$$
\begin{aligned}
m & =\sum_{i=1}^{4} m_{i}, \\
m r_{G} & =\sum_{i=1}^{4} m_{i} r_{i} \quad \text { and } \\
J_{\xi \xi}+J_{\eta \eta} & =\sum_{i=1}^{4} m_{i}\left(\eta_{i}^{2}+\xi_{i}^{2}\right),
\end{aligned}
$$

where $m$ is the mass of the rigid body, $r_{G}$ is the position vector of the centre of mass of the rigid body, $J_{\xi \xi}$ and $J_{\eta \eta}$ are the moments of inertia of the rigid body with respect to the body-attached coordinate frame, $m_{i}$ is the mass of particle $i$ and $r_{i}$ is the position 
vector of particle $i$ with respect to the body attached coordinate frame. The $4 \times 4$ linear system given by (2.1) can be put in the following matrix form:

$$
\left[\begin{array}{cccc}
1 & 1 & 1 & 1 \\
\xi_{1} & \xi_{2} & \xi_{3} & \xi_{4} \\
\eta_{1} & \eta_{2} & \eta_{3} & \eta_{4} \\
\eta_{1}^{2}+\xi_{1}^{2} & \eta_{2}^{2}+\xi_{2}^{2} & \eta_{3}^{2}+\xi_{3}^{2} & \eta_{4}^{2}+\xi_{4}^{2}
\end{array}\right]\left[\begin{array}{c}
m_{1} \\
m_{2} \\
m_{3} \\
m_{4}
\end{array}\right]=\left[\begin{array}{c}
m \\
m \xi_{G} \\
m \eta_{G} \\
J_{\xi \xi}+J_{\eta \eta}
\end{array}\right]
$$

It should be noted that, in the case of a planar rigid rod, particle 2 can be conveniently chosen to coincide with particle 4.

If the rigid body is connected to other bodies in a serial chain by revolute joints, then the primary particles 1,2 and 3 may be conveniently located at the centres of these joints. Two adjacent rigid bodies contribute to the mass concentrated at the joint connecting them. This process reduces the total number of particles replacing the whole system and leads to the automatic elimination of the constraint forces associated with the revolute joints connecting the bodies.

In general, in the case of spatial motion, a minimum number of four constrained particles, not all of which lie in the same plane, can be used to replace the rigid body. The equality of the mass, the position of the centre of mass and the six independent components of the inertia tensor result in ten conditions. To avoid the solution of nonlinear algebraic equations due to the quadratic nature of the inertia components, one may follow the same criteria suggested here and choose ten particles to obtain $10 \times 10$ linear algebraic equations in the unknown masses of the equivalent particles. Four primary particles are chosen arbitrarily, while the other six secondary particles can be conveniently located at the mid- points between the primary particles. This brings together the advantage of solving a linear system and the freedom in choosing the locations of the primary particles.

\section{Distribution of forces and couples}

Figure 2 shows a rigid body in planar motion with its equivalent system of primary particles. The positions of the three particles are given with respect to a body-attached coordinate frame. The rigid body is acted upon by an external force where the magnitude, direction and the location of its point of influence $P$ with respect to the body coordinate frame are all known. The resulting forces and their moments about any arbitrary point should sum to the original force and its moment about the same point respectively. These conditions are expressed as

$$
\sum_{i=1}^{3} f_{i}=f, \quad \sum_{i=1}^{3} S_{i} \wedge f_{i}=S^{p} \wedge f,
$$




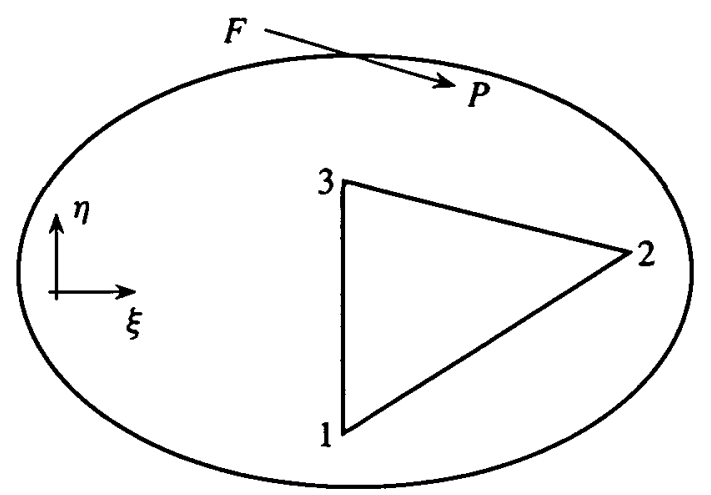

FIGURE 2. The planar rigid body with external force applied.

where $f_{i}$ is the force acting on particle $i, f$ is the external applied force, $S_{i}$ is the position vector of particle $i$ with respect to the body-attached coordinate frame, and $S^{P}$ is the position vector of the attachment point of the external force with respect to the body-attached coordinate frame. Distributing the external force over the three particles yields

$$
f_{i}=\alpha_{i} f, \quad i=1, \ldots, 3,
$$

where $\alpha_{i}$ is the distribution parameter that determines the magnitude of the force affecting particle $i$. Solving (3.1) and (3.2) for $\alpha_{i}$ gives

$$
\left[\begin{array}{lll}
1 & 1 & 1 \\
\xi_{1} & \xi_{2} & \xi_{3} \\
\eta_{1} & \eta_{2} & \eta_{3}
\end{array}\right]\left[\begin{array}{c}
\alpha_{1} \\
\alpha_{2} \\
\alpha_{3}
\end{array}\right]=\left[\begin{array}{c}
1 \\
\xi^{P} \\
\xi^{P}
\end{array}\right]
$$

In the case of a rigid rod only two parameters are required. In this case the local coordinate frame can be located with one axis coinciding with the line joining the two particles and the resulting linear system takes the form

$$
\left[\begin{array}{ll}
1 & 1 \\
\xi_{1} & \xi_{2}
\end{array}\right]\left[\begin{array}{l}
\alpha_{1} \\
\alpha_{2}
\end{array}\right]=\left[\begin{array}{c}
1 \\
\xi^{P}
\end{array}\right]
$$

Also the applied force couple is replaced analytically by an equivalent system of forces distributed over the particles. In the case of a rigid body, the applied force couple is replaced by a system of three forces affecting the lines joining the particles. The resultant of the forces should vanish and the summation of their moments should be the same as the sum of the moment of the external applied couple. For the case of a rigid rod, the applied force is replaced by two forces affecting the particles. The magnitude of the force is determined as $f=m_{c} / d$, where $m_{c}$ is the magnitude of the couple and $d$ is the distance between the two particles. 


\section{Equations of motion of a rigid body}

The rigid body shown in Figure 1 is replaced with a dynamically equivalent constrained system of three particles. The equations of motion of the resulting system of particles are derived by applying Newton's second law to study the translational motion of the four particles. For the primary particles

$$
m_{i} \ddot{r}_{i}+c_{i}=f_{i}, \quad i=1, \ldots, 3 .
$$

For the secondary particle

$$
m_{4} \ddot{r}_{4}+c_{4}=0
$$

where $m_{i}$ is the mass of particle $i, \ddot{r}_{i}$ is the acceleration vector of particle $i, f_{i}$ is the resultant of the external forces acting on particle $i$, and $f_{i}$ is the resultant of the constraint forces acting on particle $i$. The geometric constraint equations take the form

$$
\begin{array}{r}
\left(r_{1}-r_{2}\right)^{T}\left(r_{1}-r_{2}\right)-d_{1,2}^{2}=0, \\
\left(r_{1}-r_{3}\right)^{T}\left(r_{1}-r_{3}\right)-d_{1,3}^{2}=0, \\
\left(r_{2}-r_{3}\right)^{T}\left(r_{2}-r_{3}\right)-d_{2,3}^{2}=0, \\
r_{4}-\left(r_{1}+r_{3}\right) / 2=0,
\end{array}
$$

where $d_{i, j}$ is the distance between particles $i$ and $j$. Equations (4.2a)-(4.2c) are written to fix the distance between particles 1 and 2,1 and 3 , and 2 and 3 while (4.2d) locates particle 4 centrally between particles 1 and 3 . By expressing the constraint forces in terms of Lagrange multipliers and using (4.2d) to eliminate the accelerations of the secondary particle 4 , the equations of motion can be written in the following final form:

$$
\left[\begin{array}{cc}
M & -\phi_{r}^{T} \\
\phi_{r} & 0
\end{array}\right]\left[\begin{array}{l}
\ddot{r} \\
\lambda
\end{array}\right]=\left[\begin{array}{l}
f \\
\gamma
\end{array}\right]
$$

where $\ddot{r}=\left[\ddot{r}_{1}^{T}, \ddot{r}_{2}^{T}, \ddot{r}_{3}^{T}\right]^{T}$ is the unknown acceleration vector of the particles, $f=$ $\left[f_{1}^{T}, f_{2}^{T}, f_{3}{ }^{T}\right]^{T}$ is the vector of external forces distributed over the primary particles, $\phi_{r}$ is the $3 \times 6$ Jacobian matrix derived by taking the partial derivatives of the constraint equations (4.2a)-(4.2c) with respect to the Cartesian coordinates $r_{i}, i=1, \ldots, 3$, $\lambda=\left[\lambda_{1}, \lambda_{2}, \lambda_{3}\right]^{T}$ are the Lagrange multipliers associated with the constraint equations (4.2a)-(4.2c) where the constraint forces associated with these constraints can be expressed as $C=\phi_{r}^{\tau} \lambda, \gamma$ is the right-hand side of the acceleration equations derived 
by taking the time derivatives of the constraint equations (4.2a)-(4.2c) and $M$ is the $6 \times 6$ symmetric mass matrix given by

$$
M=\left[\begin{array}{cccccc}
M_{1} & 0 & 0 & 0 & m_{4} / 4 & 0 \\
0 & M_{1} & 0 & 0 & 0 & m_{4} / 4 \\
0 & 0 & m_{2} & 0 & 0 & 0 \\
0 & 0 & 0 & m_{2} & 0 & 0 \\
m_{4} / 4 & 0 & 0 & 0 & M_{3} & 0 \\
0 & m_{4} / 4 & 0 & 0 & 0 & M_{3}
\end{array}\right]
$$

Here $M_{1}=m_{1}+m_{4} / 4$ and $M_{3}=m_{3}+m_{4} / 4$.

Equation (4.3) represents a system of nine differential-algebraic equations that can be solved for the unknown accelerations of the three primary particles and the Lagrange multipliers. Similar procedures can be followed to obtain the equations of motion of a rigid rod where particles 1 and 2 are located at both ends of the rod while the third particle is located at the mid-point between the two particles. The constraint equations between the particles are given by

$$
\begin{aligned}
\left(r_{1}-r_{2}\right)^{T}\left(r_{1}-r_{2}\right)-d_{1,2}^{2} & =0, \\
r_{3}-\left(r_{1}+r_{2}\right) / 2 & =0,
\end{aligned}
$$

and by utilising (4.4b) to eliminate the acceleration of particle 3 , we obtain the equations of motion in the form given by (4.3) where $\ddot{r}=\left[\ddot{r}_{1}^{T}, \ddot{r}_{2}^{T}\right]^{T}$ is the unknown acceleration vector of the particles, $f=\left[f_{1}^{T}, f_{2}^{T}\right]^{T}$ is the vector of external forces distributed over the particles, $\phi_{r}, r=1,2$, is the $1 \times 4$ Jacobian matrix of the distance constraint (4.4a), $\lambda=\lambda_{1}$ is the Lagrange multiplier associated with the constraint equation (4.4a), $\gamma$ is the right-hand side of (4.4a), and $M$ is the $4 \times 4$ symmetric sparse mass matrix given by

$$
M=\left[\begin{array}{cccc}
M_{1} & 0 & m_{3} / 4 & 0 \\
0 & M_{1} & 0 & m_{3} / 4 \\
m_{3} / 4 & 0 & M_{2} & 0 \\
0 & m_{3} / 4 & 0 & M_{2}
\end{array}\right] .
$$

Here $M_{1}=m_{1}+m_{3} / 4$ and $M_{2}=m_{2}+m_{3} / 4$.

\section{Equations of motion of a system of rigid bodies}

A mechanical system is a collection of individual rigid bodies interconnected by kinematical joints and/or force elements. If there are no kinematical joints in the system, it is called a system of unconstrained bodies. If there are one or more 
kinematical joints in the system, it is referred to as a system of constrained bodies. In the case of an unconstrained system, the rigid bodies do not share any common particles. Then the overall mass matrix is constructed by assembling the different mass matrices for the individual bodies in the system without overlapping. The final form of the equations of motion is given by

$$
\left[\begin{array}{cc}
M & -D^{(g) T} \\
D^{(g)} & 0
\end{array}\right]\left[\begin{array}{c}
\ddot{r} \\
\lambda^{(g)}
\end{array}\right]=\left[\begin{array}{c}
g \\
\gamma^{(g)}
\end{array}\right]
$$

where $M$ is the overall mass matrix, $\ddot{r}$ represents the acceleration vectors of the primary particles, $D^{(g)}$ is the Jacobian matrix of the geometric constraints between the particles, $\lambda^{(g)}$ are the Lagrange multipliers associated with the geometric constraints between the particles, $g$ is the resultant vector of external forces acting on the primary particles, $\gamma^{(g)}$ is the right-hand side of the acceleration equations of the geometric constraints, and $(g)$ is a superscript which stands for geometric constraints.

In the case of a constrained system, two or more adjacent bodies usually share one or more particles. The mass matrices corresponding to these bodies are overlapped. The overall mass matrix is constructed by assembling the individual mass matrices and adding the contributions of the common particles. Additional kinematical constraints exist due to the kinematical joints. The final form of the equations of motion for a general constrained system is given by

$$
\left[\begin{array}{cc}
M & -D^{T} \\
D & 0
\end{array}\right]\left[\begin{array}{l}
\ddot{r} \\
\lambda
\end{array}\right]=\left[\begin{array}{l}
g \\
\gamma
\end{array}\right]
$$

Here $D$ is the Jacobian matrix of the constraints between the particles, $\lambda$ are the Lagrange multipliers associated with the constraints between the primary particles, and $\gamma$ is the right-hand side of the acceleration equations of the constraints.

\section{Equations of motion in the joint coordinates}

Derivation of the equations of motion in terms of the equivalent system of particles is simple and straightforward. The main disadvantage of this formulation is the assignment of a large number of dependent generalised coordinates which results in a large number of coupled differential-algebraic equations. The numerical solution and integration of this mixed set of equations are computationally inefficient. The equations of motion, written initially in terms of the Cartesian coordinates of the particles, are transformed to a reduced set of equations in terms of a selected set of relative joint variables. This transformation is done using a velocity transformation matrix which relates the Cartesian velocities of the particles to the relative joint velocities and allows an efficient solution and integration of the equations of motion. 
6.1. Equations of motion for an open loop system For a multibody system with open loops the equations of motion in terms of the Cartesian coordinates of the particles as given by (5.2), are transformed to a minimal set of differential equations equal to the number of degrees of freedom of the system. In the process of transformation, the position of a body is defined with respect to its adjacent reference body by relative angles or distances. Therefore the vector of joint coordinates is determined by the type of the kinematical joints. The vectors of joint coordinates, velocities and accelerations are respectively defined by

$$
\theta=\left[\theta_{1}, \ldots, \theta_{n}\right]^{T}, \quad \dot{\theta}=\left[\dot{\theta}_{1}, \ldots, \dot{\theta}_{n}\right]^{T}, \quad \ddot{\theta}=\left[\ddot{\theta}_{1}, \ldots, \ddot{\theta}_{n}\right]^{T},
$$

where $n$ is the number of degrees of freedom of the system. The Cartesian velocities of the particles are related to the joint velocities through

$$
\dot{q}=B \dot{\theta},
$$

where the matrix $B$ is the velocity transformation matrix which is a function of the Cartesian coordinates of the particles and it depends on the topology of the system as well as the type of the kinematical joints connecting the adjacent bodies. The time derivative of (6.1) gives the acceleration transformation as

$$
\ddot{q}=B \ddot{\theta}+\dot{B} \dot{\theta} .
$$

Substituting the Cartesian accelerations from (6.2) in the first relation of (5.2) and premultiplying by the matrix $B^{T}$ yields

$$
B^{T} M B \ddot{\theta}-B^{T} D^{T} \lambda=B^{T}(g-M \dot{B} \dot{\theta}) .
$$

The second term in (6.3) vanishes and the final form of the equations of motion in terms of the relative joint variables, if all the joint variables are independent, is given as

$$
\bar{M} \ddot{\theta}=f,
$$

where $\bar{M}=B^{T} M B$ and $f=B^{T}(g-M \dot{B} \dot{\theta})$. Solving the symmetric linear system of (6.4) yields the unknown joint accelerations. The estimated joint accelerations are integrated, given the initial joint coordinates and velocities, to determine the updated values of the joint coordinates and velocities.

6.2. Equations of motion for a closed loop system For multibody systems containing closed kinematical loops, additional constraint equations are introduced due to cut-joints. Each closed loop is cut at one of the kinematical joints in order to produce a reduced open loop system. For this reduced system, joint variables are defined for every open loop. The resulting equations of motion in this case are a coupled set of 
differential-algebraic equations. Let the cut-joint constraint equations for the closed kinematical loop be expressed as

$$
\psi(\theta)=0
$$

The first and second time derivatives of the constraints are given by

$$
\begin{aligned}
& \dot{\psi}=C \dot{\theta}=0, \\
& \ddot{\psi}=C \ddot{\theta}=0,
\end{aligned}
$$

where $C$ is the Jacobian matrix of the cut-joint constraints. The constraint forces associated with the cut-joint constraints are expressed in terms of Lagrange multipliers and introduced into the equations of motion for an open loop system, (6.4), to give

$$
\left[\begin{array}{cc}
\bar{M} & -C^{T} \\
C & 0
\end{array}\right]\left[\begin{array}{l}
\ddot{\theta} \\
v
\end{array}\right]=\left[\begin{array}{c}
f \\
-\dot{C} \dot{\theta}
\end{array}\right]
$$

where $v$ is the vector of Lagrange multipliers associated with the cut-joint constraints. Equations (6.5)-(6.8) represent the equations of motion for a multibody system when the number of selected joint coordinates is greater than the number of degrees of freedom of the system.

It should be noted that in this formulation, the kinematical constraints due to some common types of kinematical joints (for example, revolute or spherical joints) can be automatically eliminated by properly locating the equivalent particles. The remaining kinematical constraints along with the geometric constraints are, in general, either linear or quadratic in the Cartesian coordinates of the particles. Therefore the coefficients of their Jacobian matrix are constants or linear in the rectangular Cartesian coordinates. In the formulation based on the relative coordinates, however, the constraint equations are derived based on loop closure equations which have the disadvantage that they do not directly determine the positions of the links and points of interest which makes the establishment of the dynamic problem more difficult. Also, the resulting constraint equations are highly nonlinear and contain complex circular functions. The absence of these circular functions in the point coordinate formulation leads to faster convergence and better accuracy. Furthermore, preprocessing the mechanism by topological graph theory is not necessary, as would be the case with loop constraints.

Also, in comparison with the absolute coordinate formulation, the manual work of the local axes attachment and local coordinate evaluation as well as the use of the rotational variables and the rotation matrices in the absolute coordinate formulation are not required in the point coordinate formulation. This leads to fully computerised analysis and accounts for a reduction in the computational time and memory storage. 
In addition to that, the constraint equations take much simpler forms as compared with the absolute coordinates. Furthermore, the use of absolute coordinates may cause numerical problems if differences of large values of the absolute coordinates are used, for example, for the calculation of spring or damper forces or constraint residuals.

The elimination of the rotational coordinates, in the presented formulation, leads to possible savings in computation time when this procedure is compared against the absolute or relative coordinate formulation. It has been determined that numerical computations associated with rotational transformation matrices and their corresponding coordinate transformations between reference frames is time consuming and, therefore, if these computations are avoided more efficient codes may be developed. The elimination of rotational coordinates can also be found to be very beneficial in design sensitivity analysis of multibody systems. In most procedures for design sensitivity analysis, leading to an optimal design process, the derivatives of certain functions with respect to a set of design parameters are required. Analytical evaluation of these derivatives are much simpler if the rotational coordinates are not present and if we only deal with translational coordinates.

Some practical applications of multibody dynamics require one or more bodies in the system to be described as deformable in order to obtain a more realistic dynamic response. Deformable bodies are normally modelled by the finite element technique. Assume that the deformable body is connected to a rigid body described by a set of particles. Then one or more particles of the rigid body can coincide with one or more nodes of the deformable body in order to describe the kinematical joint between the two bodies. This is a much simpler process than when the rigid body is described by a set of translational and rotational coordinates. In general, the point coordinates have additional advantages over the other systems of coordinates since they are the most suitable coordinates for graphics routines and for animation programs.

6.3. Integration of the equations of motion The differential equations of motion for an open-chain, (6.4), or for a closed-chain, (6.8), represent a set of nonlinear ordinary differential equations with time as an independent variable. The system can be put in the standard form $\dot{y}=f(y, t)$, where $y$ and $\dot{y}$ are the vectors that contain the relative joint coordinates, velocities and accelerations as

$$
\dot{y}=\left[\begin{array}{l}
\dot{\theta} \\
\ddot{\theta}
\end{array}\right] \text { and } y=\left[\begin{array}{c}
\theta \\
\dot{\theta}
\end{array}\right] \text {. }
$$

The numerical solution of the equations of motion requires a numerical integration process that determines the elements of $y$ at every time step. The function $f$ is evaluated by solving the equations of motion for the unknown joint accelerations. This numerical process is summarised as follows: 
(1) Initially, the joint coordinates and velocities are known, that is, $y_{0}=\left[\theta_{0}^{T}, \dot{\theta}_{0}^{T}\right]^{T}$.

(2) Using the vector $y_{0}=\left[\theta_{0}^{T}, \dot{\theta}_{0}^{T}\right]^{T}$, the Cartesian coordinates and velocities and hence the matrices $B, \dot{B}, C$ and $\dot{C}$ can be constructed.

(3) With knowledge of the known constant mass matrix $M$ and the force vector $f$, the equations of motion for an open-chain, (6.4), or a closed-chain, (6.8), can be derived.

(4) Solve the equations of motion for $\ddot{\theta}$ and $\nu$ using the Gaussian elimination technique adopted for symmetric matrices.

(5) Construct the $\dot{y}$ vector and return the contents to the integration algorithm.

(6) Repeat the previous steps at every time step.

Gear's method [1] for the numerical integration of differential-algebraic equations is used to overcome the instability problem resulting during the modelling process of constrained mechanical systems. Use of both Cartesian and joint coordinates produces an efficient set of equations without loss of generality.

\section{Dynamic analysis of a closed-chain}

The planar three degree-of-freedom platform-type manipulator shown in Figure 3 is chosen as an example of a closed-chain. The end-effector platform of the manipulator is constrained by three serial link trains, each of which possesses three revolute joints which form two independent closed loops. Table 1 presents the mass and the mass distribution over the links of the manipulator. Each rigid link is replaced by an

TABLE 1. Description of rigid bodies

\begin{tabular}{|c|c|c|}
\hline Body & Mass $(\mathrm{kg})$ & Inertia $J_{\xi \xi}+J_{\eta \eta}\left(\mathrm{kg} \mathrm{m}^{2}\right)$ \\
\hline Rigid rods & 1.0 & 0.333333 \\
\hline platform & 3.0 & 1.0 \\
\hline
\end{tabular}

equivalent system of two primary particles located at both ends and an intermediate secondary particle. The rigid platform is replaced by a system of three primary particles located at joints connecting them to the other links and a secondary particle at the middle of any pair of them. Locating the particles belonging to adjacent bodies together at the connection joints reduces the total number of particles replacing the whole system and leads to the automatic elimination of the kinematical constraints at these joints. As shown in Figure 3, an overall equivalent system of nine primary particles is constructed and a resultant $16 \times 16$ sparse symmetric mass matrix is constructed. Since two closed loops exist, two joints should be cut. Cutting the revolute joints at points 7 and 9 produces two open-chains. Then the vector of joint coordinates and the corresponding vectors of joint velocities and accelerations are, 


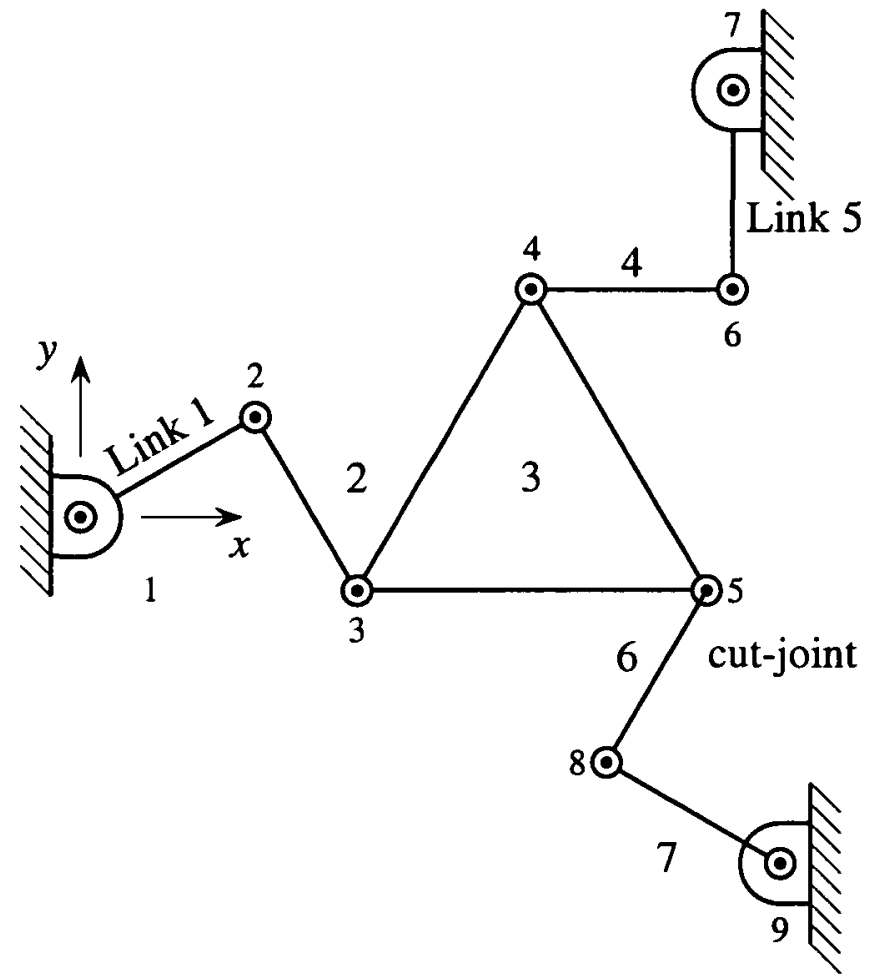

FIGURE 3. The manipulator with the equivalent particles.

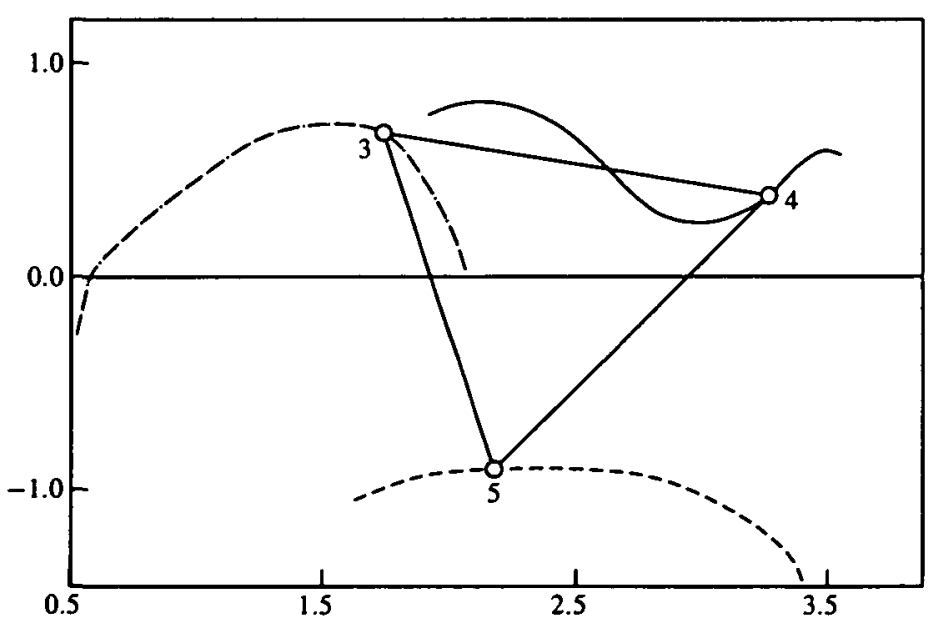

FIGURE 4. The trajectory of the platform. 
respectively, defined by

$$
\begin{aligned}
& \theta=\left[\theta_{1}, \theta_{2,1}, \theta_{3,2}, \theta_{4,3}, \theta_{5,4}, \theta_{6,3}, \theta_{7,6}\right]^{T}, \\
& \dot{\theta}=\left[\dot{\theta}_{1}, \dot{\theta}_{2,1}, \dot{\theta}_{3,2}, \dot{\theta}_{4,3}, \dot{\theta}_{5,4}, \dot{\theta}_{6,3}, \dot{\theta}_{7,6}\right]^{T}, \\
& \ddot{\theta}=\left[\ddot{\theta}_{1}, \ddot{\theta}_{2,1}, \ddot{\theta}_{3,2}, \ddot{\theta}_{4,3}, \ddot{\theta}_{5,4}, \ddot{\theta}_{6,3}, \ddot{\theta}_{7,6}\right]^{T},
\end{aligned}
$$

where $\theta_{1}$ is the inclination angle of body 1 with the horizontal, and $\theta_{j, i}$ is the relative angle between bodies $j$ and $i$. The velocity transformation equation has the form

$$
\left[\begin{array}{c}
\dot{r}_{2} \\
\dot{r}_{3} \\
\dot{r}_{4} \\
\dot{r}_{5} \\
\dot{r}_{6} \\
\dot{r}_{7} \\
\dot{r}_{8} \\
\dot{r}_{9}
\end{array}\right]=\left[\begin{array}{lllll}
k \wedge r_{2,1} & & & & \\
k \wedge r_{3,1} & k \wedge r_{3,2} & & & \\
k \wedge r_{4,1} & k \wedge r_{4,2} & k \wedge r_{4,3} & & \\
k \wedge r_{5,1} & k \wedge r_{5,2} & k \wedge r_{5,3} & & \\
k \wedge r_{6,1} & k \wedge r_{6,2} & k \wedge r_{6,3} & k \wedge r_{6,4} & \\
k \wedge r_{7,1} & k \wedge r_{7,2} & k \wedge r_{7,3} & k \wedge r_{7,4} & k \wedge r_{7,6} \\
k \wedge r_{8,1} & k \wedge r_{8,2} & k \wedge r_{8,3} & k \wedge r_{8,5} & \\
k \wedge r_{9,1} & k \wedge r_{9,2} & k \wedge r_{9,3} & k \wedge r_{9,5} & k \wedge r_{9,8}
\end{array}\right]\left[\begin{array}{c}
\dot{\theta}_{1} \\
\dot{\theta}_{2,1} \\
\dot{\theta}_{3,2} \\
\dot{\theta}_{4,3} \\
\dot{\theta}_{5,4} \\
\dot{\theta}_{6,3} \\
\dot{\theta}_{7,6}
\end{array}\right]
$$

Two cut-joint constraint equations are written to prevent the motion of particles 7 and 9 , in vector form as $r_{7}-c_{1}=0$ and $r_{9}-c_{2}=0$, where $c_{1}$ and $c_{2}$ are known constant vectors. The corresponding velocity and acceleration equations take, respectively, the form $\dot{r}_{7}-\dot{r}_{9}=0$ and $\ddot{r}_{7}-\ddot{r}_{9}=0$.

The $4 \times 5$ matrix $C$, defined in (6.6), can be put in the following closed form:

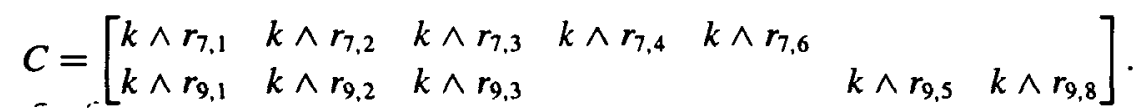

The resulting equations of motions, as given by (6.8), represent an $11 \times 11$ symmetric linear system that can be solved for the unknown joint accelerations and reaction forces at the cut joints. The motion of the system is started from the rest position under the action of gravity forces as well as an external force of magnitude $10 \mathrm{~N}$ applied at the centre of the end-effector in the horizontal direction. Figure 4 presents the trajectory of the platform and indicates the oscillating behaviour controlled by the gravitational and constraint forces. Verification of the results is done by comparison with the absolute coordinate formulation [4].

\section{Conclusion}

In this paper a two-step formulation for the dynamic analysis of generalised planar linkages is developed. Initially a rigid body is replaced by a dynamically equivalent constrained system of particles and the equations of motion are formulated using 
only Newton's second law in terms of the Cartesian coordinates of the particles. The simplicity and the absence of any rotational coordinates and the corresponding transformation matrices from the final form of the equations of motion are considered the main advantages of this formulation. Then the equations of motion are transformed to a reduced set using relative joint coordinates. This leads to an efficient solution and integration of the equations of motion. The formulation is then applied to carry out the dynamic analysis of a closed-chain. The simulation demonstrates the generality and efficiency of the formulation.

\section{References}

[1] C. W. Gear, "Differential-algebraic equations index transformations", SIAM J. Sci. Statist. Comput. 9 (1988) 39-47.

[2] J. E. Keat and J. D. Turner, "Equations of motion of multibody systems for applications to large space structure development", in AIAA Dynamics Specialist Conference, Palm Springs, CA, AIAA Paper 84-1014-CP, (1984).

[3] S. S. Kim and M. J. Vanderploeg, "A general and efficient method for dynamic analysis of mechanical systems using velocity transformation", ASME J. Mechanisms, Transmissions and Automation in Design 108 (1986) 176-182.

[4] P. E. Nikravesh, Computer aided analysis of mechanical systems (Prentice-Hall, Englewood Cliffs, NJ, 1988).

[5] P. E. Nikravesh and G. Gim, "Systematic construction of the equations of motion for multibody systems containing a closed kinematic loop", in Proceedings of the ASME Design Conference, (1989).

[6] N. Orlandea, M. A. Chace and D. A. Calahan, "A sparsity-oriented approach to dynamic analysis and design of mechanical systems, Part I and II", ASME J. Engineering for Industry 99 (1977) 773-784.

[7] B. Paul and D. Krajcinovic, "Computer analysis of machines with planar motion. 1. Kinematics, 2. Dynamics", ASME J. Appl. Mech. 37 (1970) 697-712.

[8] W. O. Schiehlen, Multibody systems handbook (Springer, Berlin, 1990).

[9] W. O. Schiehlen, "Multibody system dynamics: Roots and perspectives", J. Multibody System Dynamics 1 (1997) 149-188.

[10] A. A. Shabana, Computational Dynamics (John Wiley \& Sons, New York, 1994).

[11] A. A. Shabana, "Flexible multibody dynamics: Review of past and recent developments", J. Multibody System Dynamics 1 (1997) 189-222.

[12] J. Wittenburg, "Nonlinear equations of motion for arbitrary systems of interconnected rigid bodies", in Proceedings of the Symposium on Dynamics of Multibody Systems, Munich, Germany, Aug 28Sep 3, (1977). 